\title{
TWO DISTANT HALO VELOCITY GROUPS DISCOVERED BY THE PALOMAR TRANSIENT FACTORY
}

\author{
Branimir Sesar ${ }^{1}$, Judith G. Cohen ${ }^{1}$, David Levitan ${ }^{1}$, Carl J. Grillmair ${ }^{2}$, Mario Jurić ${ }^{3,4}$, Evan N. Kirby ${ }^{1,6}$, \\ Russ R. Laher ${ }^{2}$, Eran O. OfeK ${ }^{5}$, Jason A. Surace ${ }^{2}$, Shrinivas R. Kulkarni ${ }^{1}$, and Thomas A. Prince ${ }^{1}$ \\ ${ }^{1}$ Division of Physics, Mathematics and Astronomy, California Institute of Technology, Pasadena, CA 91125, USA; bsesar@ astro.caltech.edu \\ ${ }^{2}$ Spitzer Science Center, California Institute of Technology, Pasadena, CA 91125, USA \\ ${ }^{3}$ Large Synoptic Survey Telescope Corp., 933 North Cherry Avenue, Tucson, AZ 85721, USA \\ ${ }^{4}$ Steward Observatory, University of Arizona, Tucson, AZ 85721, USA \\ ${ }^{5}$ Benoziyo Center for Astrophysics, Weizmann Institute of Science, 76100 Rehovot, Israel \\ Received 2012 March 7; accepted 2012 June 26; published 2012 August 2
}

\begin{abstract}
We report the discovery of two new halo velocity groups (Cancer groups A and B) traced by eight distant RR Lyrae stars and observed by the Palomar Transient Factory survey at R.A. $\sim 129^{\circ}$, decl. $\sim 20^{\circ}\left(l \sim 205^{\circ}, b \sim 32^{\circ}\right)$. Located at $92 \mathrm{kpc}$ from the Galactic center $(86 \mathrm{kpc}$ from the Sun), these are some of the most distant substructures in the Galactic halo known to date. Follow-up spectroscopic observations with the Palomar Observatory $5.1 \mathrm{~m}$ Hale telescope and W. M. Keck Observatory $10 \mathrm{~m}$ Keck I telescope indicate that the two groups are moving away from the Galaxy at $\bar{v}_{\text {gsr }}^{A}=78.0 \pm 5.6 \mathrm{~km} \mathrm{~s}^{-1}$ (Cancer group A) and $\bar{v}_{\text {gsr }}^{B}=16.3 \pm 7.1 \mathrm{~km} \mathrm{~s}^{-1}$ (Cancer group B). The groups have velocity dispersions of $\sigma_{v_{\mathrm{gsr}}}^{A}=12.4 \pm 5.0 \mathrm{~km} \mathrm{~s}^{-1}$ and $\sigma_{v_{\mathrm{gsr}}}^{B}=14.9 \pm 6.2 \mathrm{~km} \mathrm{~s}^{-1}$ and are spatially extended (about several kpc), making it very unlikely that they are bound systems, and more likely to be debris of tidally disrupted dwarf galaxies or globular clusters. Both groups are metal-poor (median metallicities of $[\mathrm{Fe} / \mathrm{H}]^{\mathrm{A}}=-1.6 \mathrm{dex}$ and $[\mathrm{Fe} / \mathrm{H}]^{\mathrm{B}}=-2.1 \mathrm{dex}$ ) and have a somewhat uncertain (due to small sample size) metallicity dispersion of $\sim 0.4 \mathrm{dex}$, suggesting dwarf galaxies as progenitors. Two additional RR Lyrae stars with velocities consistent with those of the Cancer groups have been observed $\sim 25^{\circ}$ east, suggesting possible extension of the groups in that direction.
\end{abstract}

Key words: Galaxy: halo - Galaxy: kinematics and dynamics - Galaxy: structure - stars: variables: RR Lyrae

Online-only material: color figures

\section{INTRODUCTION}

In the last decade, deep and wide-area astronomical surveys, such as the Sloan Digital Sky Survey (SDSS; York et al. 2000) and Two Micron All Sky Survey (2MASS; Skrutskie et al. 2006), have enabled detection of substructures in the halo both as stellar overdensities in space and as moving groups. The tidal streams of the disrupting Sagittarius dwarf galaxy (Ibata et al. 1994) are the best example of such substructures, with streams wrapping around most of the sky (Majewski et al. 2003). Other known substructures include the Pisces Overdensity ("Clump J" in Sesar et al. 2007; Watkins et al. 2009; Sesar et al. 2010a), the GD-1 stream (Grillmair \& Dionatos 2006), the Orphan stream (Grillmair 2006; Belokurov et al. 2007), and several other overdensities and streams. ${ }^{7}$

While many halo substructures have been discovered so far, the motivation for finding and characterizing more of them remains strong. For example, at larger galactocentric distances $\left(R_{\mathrm{GC}} \gtrsim 15 \mathrm{kpc}\right)$, the Galactic gravitational potential is dominated by dark matter and observations of more distant tidal streams can be used to constrain the shape, orientation, and mass of the Milky Way's dark matter halo (e.g., Law \& Majewski 2010; Watkins et al. 2010). Other motivations for completing the census of halo substructures are to better constrain the severity of the so-called missing satellite problem (Klypin et al. 1999; Moore et al. 1999) and the contribution of the accretion of dwarf satellite galaxies to the formation of the Galactic halo.

\footnotetext{
${ }^{6}$ Hubble Fellow.

7 A fairly extensive list of halo streams and overdensities can be found at http://homepages.rpi.edu/ newbeh/mwstructure/ MilkyWaySpheroidSubstructure.html
}

Finding distant halo substructures, however, is not an easy task. One approach to this problem is to look for spatial groups of (non-variable) sources that are consistent in color-magnitude space with, for example, an old population of stars at a fixed distance (e.g., Willman et al. 2005; Grillmair 2006; Belokurov et al. 2007). While this technique has been employed quite successfully in the past, its strongest limitation when looking for distant halo substructures using ground-based imaging data is the separation of stars from galaxies at faint magnitudes. At magnitudes fainter than $r \sim 21$ (corresponding to a heliocentric distance of $\sim 30 \mathrm{kpc}$ for metal-poor main-sequence turn-off stars), the number of field Milky Way stars per unit magnitude decreases with increasing magnitude (i.e., the number density profile of Galactic halo stars steepens with distance from the Galactic center from a power law with index $n=-2.6$ to that with $n \lesssim-3.8$; Sesar et al. 2011; Deason et al. 2011), while the number of galaxies per unit magnitude increases. In addition, morphological separation of stars and galaxies (e.g., SDSS resolved-unresolved source classification; Lupton et al. 2002) becomes increasingly unreliable toward faint magnitudes as the signal-to-noise ratio $(\mathrm{S} / \mathrm{N})$ decreases. The increasing contribution of galaxies in point-source catalogs at faint magnitudes causes a sharp increase in the noise from which the signal of a true low surface brightness stellar system must be detected.

Instead of looking for clustering in samples of non-variable tracers, which may get contaminated by galaxies at faint magnitudes, we should look for clustering in samples of variable tracers with distinctive light curves (e.g., RR Lyrae stars) that are difficult to confuse with galaxies. RR Lyrae stars have several advantages when used to map the Galactic halo because they (1) are old, evolved stars and therefore trace old stellar populations (Smith 1995); (2) have distinct light curves, which 
make them easy to identify given multi-epoch observations (peak-to-peak amplitudes of $V \sim 1 \mathrm{mag}$ and periods of $\sim 0.6$ days); and (3) are bright, standard candles $\left(M_{V}=0.6 \mathrm{mag}\right.$ at $[\mathrm{Fe} / \mathrm{H}]=-1.5 \mathrm{dex}$, with $\sim 7 \%$ uncertainty in distance) that can be detected at large distances $(5-120 \mathrm{kpc}$ for $14<V<21)$. The steepening of the stellar density profile beyond $30 \mathrm{kpc}$ is actually beneficial for searches that employ RR Lyrae stars because it reduces the pool of stars that can form false spatial groups and therefore increases the contrast between stars associated with halo substructures and stars associated with the smooth halo (e.g., the Pisces Overdensity shown in Figure 11 of Sesar et al. 2010a). Due to the steepening of the stellar density profile beyond $30 \mathrm{kpc}$, groups of distant RR Lyrae stars are more likely to be real halo substructures than chance associations of stars. While the probability of chance association at large distances and small angular scales is very small, it is not zero and spectroscopic follow-up is still needed to test whether the stars in a spatial group also form a moving group.

In this paper, we use the line of reasoning presented above and successfully used by Kollmeier et al. (2009) and Sesar et al. (2010b) as a motivation to study a group of eight RR Lyrae stars found during a preliminary search for halo substructures in regions of the sky observed by the Palomar Transient Factory (PTF) survey. ${ }^{8}$ The data set and the algorithm used to select RR Lyrae stars are described in Sections 2 and 3. The spectroscopic observations, their reduction, and measurement of line-of-sight velocities and metallicities are described in Section 5. The results are discussed in Section 6, and our conclusions are presented in Section 7.

\section{OVERVIEW OF THE PALOMAR TRANSIENT FACTORY SURVEY}

The PTF (Law et al. 2009; Rau et al. 2009) is a synoptic survey designed to explore the transient sky. The project utilizes the 48 inch Samuel Oschin Schmidt Telescope on Mount Palomar. The telescope has a digital camera equipped with 12 CCDs (one of which is not active), each $2 \mathrm{~K} \times 4 \mathrm{~K}$ pixels (Rahmer et al. 2008). Each PTF image covers $7.26 \mathrm{deg}^{2}$ with a pixel scale of 1 ". 01 . By the end of 2011, the PTF observed $\sim 8000 \mathrm{deg}^{2}$ of sky at least 30 times in the Mould- $R$ filter ${ }^{9}$ (hereafter, the $R$-band filter) and $\sim 1800 \mathrm{deg}^{2}$ in the SDSS $g^{\prime}$ filter.

Some regions of the sky, known as the "high-cadence" regions in PTF, have been observed more than 100 times. One such region on which we focus in this paper is a $45 \mathrm{deg}^{2}$ field roughly centered on the Praesepe ${ }^{10}$ cluster $\left(08^{\mathrm{h}} 40^{\prime} 24^{\prime \prime},+19^{\circ} 41^{\prime}\right)$, which has been imaged more than 200 times by PTF in the $R$ band (Agüeros et al. 2011; Polishook et al. 2012). Relative to other, less observed areas, the Praesepe region is interesting because, due to chance and a large number of observations, it has a nonnegligible number of images that are deeper than the nominal $5 \sigma$ faint limit at $R=20.6$ (i.e., images that were taken in better-than-average conditions). In turn, these deeper images increase the likelihood of finding distant RR Lyrae stars and halo substructures, if any are present.

We use PTF data processed by the photometric pipeline, which is hosted by the Infrared Processing and Analysis Center. This pipeline performs final image reduction, source extraction, and photometric and astrometric calibration (Grillmair et al.

\footnotetext{
8 http://www.astro.caltech.edu/ptf

9 The PTF Mould- $R$ filter is similar in shape to the SDSS $r$-band filter, but shifted $27 \AA$ redward.

10 Also known as the Beehive Cluster and M44.
}

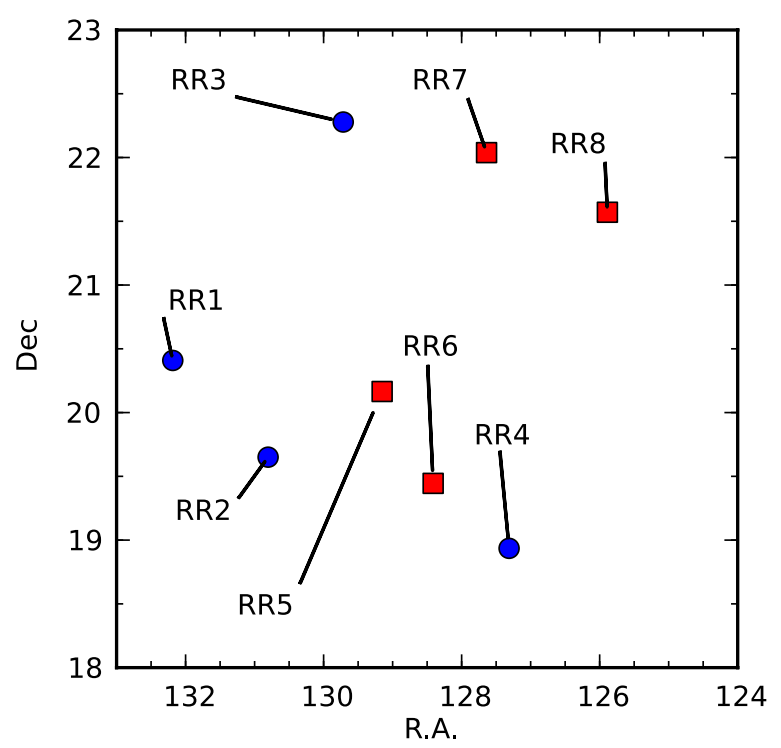

Figure 1. Spatial distribution of eight $a b$-type RR Lyrae stars studied in this work, which are located at distances of 77-96 kpc from the Sun (84-102 kpc from the Galactic center). Their positions and light-curve parameters are listed in Table 1. The symbols indicate the halo velocity group membership for each of these stars in either the $\bar{v}_{\mathrm{gsr}} \sim 80 \mathrm{~km} \mathrm{~s}^{-1}$ group (blue circles) or the $\bar{v}_{\text {gsr }} \sim 15 \mathrm{~km} \mathrm{~s}^{-1}$ group (red squares, see Section 6). The lines simply connect labels and symbols. According to the completeness analysis presented in Section 4, these are the only RRab stars in this region of the Galactic halo.

(A color version of this figure is available in the online journal.)

2010; Ofek et al. 2012; R. Laher et al. 2012, in preparation). The photometric uncertainty provided by this pipeline is smaller than $\sim 0.01 \mathrm{mag}$ for $R<16$ sources and increases to $0.2 \mathrm{mag}$ at $R=$ 20.6. The algorithm used for photometric calibration is based on the one presented in Honeycutt (1992) and modifications in Ofek et al. (2011) and Levitan et al. (2011). Relative to the reference UCAC-3 astrometric catalog (Zacharias et al. 2010), the astrometric precision of PTF coordinates is about $0^{\prime} .1$ in right ascension and declination. To enable fast processing and manipulation, the data used in this paper were stored in the Large Survey Database (LSD ${ }^{11}$ ) format (Jurić 2011).

\section{SELECTION OF RR LYRAE STARS}

In Figure 1, we show the spatial distribution of eight distant (galactocentric distance $R_{\mathrm{GC}}>84 \mathrm{kpc}$ ) $a b$-type RR Lyrae (RRab) stars observed by PTF in the Praesepe region. These stars were selected during a preliminary search for RR Lyrae stars in the available PTF observations. The selection procedure we used is similar to the one outlined by Sesar et al. (2010a) and is briefly described below. The completeness of this sample is estimated in Section 4.

First, we searched for all PTF sources positionally associated within 1".5 of point-like sources in the SDSS Data Release 8 catalog (Aihara et al. 2011) that have single-epoch SDSS colors consistent with colors of RR Lyrae stars (Equations (6)-(9) from Sesar et al. 2010a):

$$
0.75<u-g<1.45
$$

$$
-0.25<g-r<0.4
$$

\footnotetext{
11 http://lsddb.org
} 
Table 1

Positions and Light-curve Parameters of RR Lyrae Targets

\begin{tabular}{|c|c|c|c|c|c|c|c|c|c|}
\hline $\mathrm{ID}^{\mathrm{a}}$ & IAU Name & $\begin{array}{l}\text { R.A. }^{\text {b }} \\
\text { (deg) }\end{array}$ & $\begin{array}{c}\text { Decl. }^{b} \\
\text { (deg) }\end{array}$ & $\begin{array}{c}\text { Distance }^{c} \\
\quad(k p c)\end{array}$ & $\begin{array}{c}A_{R}^{\mathrm{d}} \\
(\mathrm{mag})\end{array}$ & $\begin{array}{c}R_{0}{ }^{\mathrm{e}} \\
(\mathrm{mag})\end{array}$ & $\begin{array}{c}\text { Period }^{\mathrm{f}} \\
\text { (d) }\end{array}$ & $\begin{array}{c}\mathrm{HJD}_{0}{ }^{\mathrm{g}} \\
\text { (d) }\end{array}$ & $N_{\text {obs }}{ }^{\mathrm{h}}$ \\
\hline RR1 & PTF1J084844.61+202430.8 & 132.185880 & 20.408611 & $87.1 / 93.2$ & 0.887 & 19.84 & 0.515257 & 55303.686299 & 291 \\
\hline RR2 & PTF1J084313.05+193901.1 & 130.804370 & 19.650306 & $78.2 / 84.4$ & 0.889 & 19.81 & 0.556328 & 55271.737376 & 414 \\
\hline RR3 & PTF1J083851.75+221641.5 & 129.715656 & 22.278200 & $85.9 / 92.2$ & 0.766 & 19.82 & 0.555729 & 55590.910809 & 160 \\
\hline RR4 & PTF1J082914.96+185607.4 & 127.312372 & 18.935392 & $86.0 / 92.4$ & 0.629 & 20.01 & 0.594511 & 55667.607592 & 193 \\
\hline RR5 & PTF1J083636.44+200954.9 & 129.151846 & 20.165259 & $96.0 / 102.3$ & 0.855 & 19.92 & 0.610057 & 55594.666378 & 406 \\
\hline RR7 & PTF1J083033.30+220217.9 & 127.638788 & 22.038294 & $77.0 / 83.5$ & 0.703 & 19.64 & 0.545361 & 55594.799336 & 400 \\
\hline RR8 & PTF1J082333.11+213414.6 & 125.887964 & 21.570710 & $84.2 / 90.8$ & 0.912 & 19.96 & 0.517656 & 55542.999049 & 133 \\
\hline RR9 & PTF1J103051.69+202330.8 & 157.715375 & 20.391878 & $75.5 / 79.3$ & 0.914 & 19.35 & 0.544278 & 55515.539757 & 82 \\
\hline RR10 & PTF1J102040.66+212315.1 & 155.169417 & 21.387514 & $76.1 / 80.2$ & 0.759 & 19.52 & 0.611088 & 55244.324620 & 96 \\
\hline AV Peg & AV Peg & 328.011644 & 22.574827 & $0.7 / 7.9$ & 1.040 & n.a. & 0.390381 & 55833.513400 & n.a. \\
\hline
\end{tabular}

Notes. The first eight RR Lyrae stars are located in the Cancer region, the next two are offset by $\sim 25^{\circ}$ east from the Cancer region and suggest an eastward extension of Cancer groups, and AV Peg is a known RR Lyrae star used for validation of various procedures used in this work.

a Short name.

b Equatorial J2000.0 right ascension and declination from SDSS DR8 catalog.

${ }^{\mathrm{c}}$ Heliocentric/galactocentric distance calculated using spectroscopic metallicity.

${ }^{\mathrm{d}}$ Light-curve amplitude in the Mould- $R$ band ( $V$-band amplitude for AV Peg).

${ }^{\mathrm{e}} R$-band magnitude at the epoch of maximum brightness.

${ }^{\mathrm{f}}$ Period of pulsation.

g Reduced Heliocentric Julian Date of maximum brightness (HJD -2,400,000).

${ }^{\mathrm{h}}$ Number of observations in the Mould- $R$ band.

$$
\begin{aligned}
& -0.2<r-i<0.2 \\
& -0.3<i-z<0.3 .
\end{aligned}
$$

In the above equations, SDSS colors have been corrected for interstellar medium extinction using the dust map created by Schlegel et al. (1998).

Variable sources were selected from this color-selected sample by requiring that PTF $R$-band light curves have rms scatter greater than $0.1 \mathrm{mag}$ and $\chi^{2}$ per degree of freedom greater than 3 (calculated with respect to the mean magnitude associated with a given source). The five most likely periods of variability were obtained for each variable PTF source by running an implementation of the Supersmoother period-finding algorithm (Friedman 1984; Reimann 1994) on the source's $R$-band light curve. If Supersmoother returned one or more periods in the 0.2-0.9 day range (typical of RR Lyrae stars; Smith 1995), then the variable source's light curve was phased (period-folded) with each period and SDSS $r$-band RR Lyrae light-curve templates constructed by Sesar et al. (2010a) were fitted to phased data.

The light-curve fitting was performed to minimize the robust goodness-of-fit cost function defined in Equation (5) in the leastsquares sense, with the epoch of the peak brightness $\phi_{0}$, peak-topeak amplitude $A_{R}$, and peak brightness $R_{0}$ as free parameters. The quality of a template fit was defined with a $\chi^{2}$-like parameter

$$
\zeta=\operatorname{median}\left(\left|m_{\text {observed }}^{i}-m_{\text {template }}\right| / \epsilon_{\text {observed }}^{i}\right),
$$

where $m_{\text {observed }}$ and $\epsilon_{\text {observed }}$ are the observed $R$-band magnitude and its uncertainty, $m_{\text {template }}$ is the magnitude predicted by the template, and $i=1, N_{\mathrm{obs}}$, where $N_{\mathrm{obs}}$ is the number of observations. Here, we used the median to minimize the bias in $\zeta$ due to rare observations with unreliable (non-Gaussian) errors (e.g., due to image artifacts, cosmic rays). The template with the lowest $\zeta$ value was selected as the best fit, and the best-fit parameters were stored. Finally, we visually inspected template-fitted light curves to tag sources as RR Lyrae stars.

The positions and light-curve parameters of RR Lyrae stars observed in this work are listed in Table 1, and examples of phased $R$-band light curves are shown in Figure 2. The periods and epochs of maximum brightness are uncertain to within a few seconds and minutes, respectively, allowing the phase of pulsation to be determined with better than $1 \%$ uncertainty. An accurate estimate of phase is important when subtracting the velocity due to pulsations from the measured radial velocity, as we explain in Section 5.3. In addition to eight RR Lyrae stars discussed in this work (RR1 to RR8), Table 1 contains two RR Lyrae stars that were spectroscopically followed up after initial submission of this paper (RR9 and RR10). These two stars were not used in the analysis presented below. However, their position and velocities are mentioned in Section 7, as these stars may indicate an eastward extension of the Cancer groups.

The heliocentric distances of RRLyrae stars, $d$, are estimated from measurements of their flux-averaged and extinctioncorrected $R$-band magnitudes, $\langle R\rangle$, and by assuming that $M_{R} \approx$ $M_{V}$. The $M_{R} \approx M_{V}$ approximation is uncertain at $\sim 0.04 \mathrm{mag}$ level, as estimated from the rms scatter of the difference of flux-averaged $r$-and $V$-band magnitudes of RR Lyrae stars from Sesar et al. (2010a, see their Table 3). The absolute magnitudes of RRab stars in the Johnson $V$ band were calculated using the Chaboyer (1999) $M_{V}-[\mathrm{Fe} / \mathrm{H}]$ relation

$$
M_{V}=(0.23 \pm 0.04)[\mathrm{Fe} / \mathrm{H}]+(0.93 \pm 0.12),
$$

where $[\mathrm{Fe} / \mathrm{H}]$ is the spectroscopic metallicity measured in Section 5.4. The fractional error in the heliocentric distance due to uncertainty in Equation (6), spectroscopic metallicities, and RR Lyrae evolution off the zero-age horizontal branch is estimated at $7 \%(\sim 6 \mathrm{kpc}$ error in distance at $86 \mathrm{kpc})$. The apparent $\langle R\rangle$ magnitudes of the observed stars range from $19.9 \mathrm{mag}$ to $20.3 \mathrm{mag}$, corresponding to heliocentric distances of $77 \mathrm{kpc}$ and $96 \mathrm{kpc}$, respectively. 


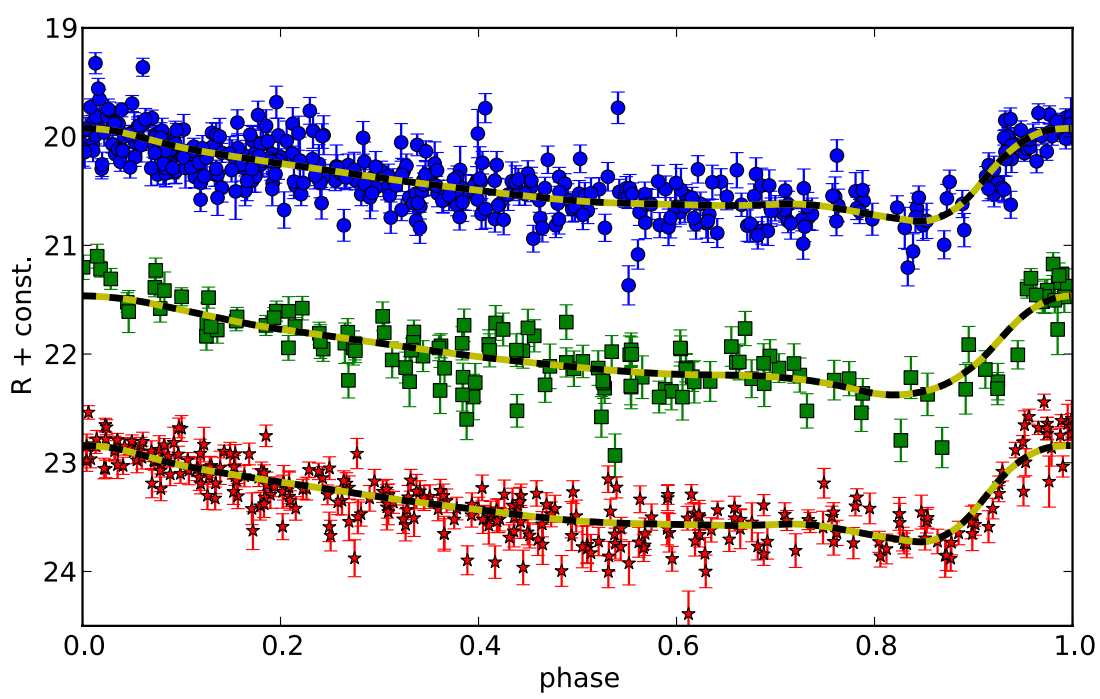

Figure 2. Phased $R$-band light curves of three RRab stars discussed in this work (from top to bottom: RR5, RR8, and RR1). The light curves are offset for clarity (offsets of $0,1.5$, and $3 \mathrm{mag}$ from top to bottom), and the solid lines show best-fit SDSS $r$-band templates constructed by Sesar et al. (2010a).

(A color version of this figure is available in the online journal.)

\section{COMPLETENESS OF THE RR LYRAE SAMPLE}

The selection procedure described in the previous section yielded eight RRab stars at distances from 77 to $96 \mathrm{kpc}$ from the Sun in the $45 \mathrm{deg}^{2}$ region of the sky centered on the Praesepe cluster (see Figure 1). The question we now pose is whether these are all the RRab stars that could have been detected in this distance range given the available PTF data. To answer this question, we need to know the success rate of our period-finding algorithm as the period estimation is the most critical step in the selection of RR Lyrae stars. If the period of pulsation is not correctly estimated, the phased light curve will not be identified as that of an RR Lyrae star.

To estimate the success rate of our period-finding algorithm, we have simulated 100 time series by randomly sampling 133 data points from a template RRab light curve with a maximum brightness $R_{0}=20 \mathrm{mag}\left(R_{0}\right.$ of the faintest $\mathrm{RRab}$ star in our sample), amplitude $A_{R}=0.3 \mathrm{mag}$, and period of 0.58 days. Random sampling of a template light curve is a reasonable choice since the observed light curves are almost uniformly sampled (see Figure 2). The amplitude of $A_{R}=0.3 \mathrm{mag}$ represents the lower range of amplitudes that RRab stars can have (e.g., see Figure 16 in Sesar et al. 2010a), and the number of data points corresponds to the number of observations of the least observed RRab star in our sample (see Table 1). To make the light curves realistic, we added Gaussian noise to sampled magnitudes using a model that describes the mean photometric uncertainty in the PTF $R$ band as a function of magnitude. We then ran mock time series through our periodfinding algorithm and counted time series where the estimated period was within $1 \%$ of the true period.

Even for this worst-case scenario, we find that the periods are successfully recovered in better than $95 \%$ of cases and conclude that our selection procedure has recovered almost every RRab star in the Praesepe region between 77 and $96 \mathrm{kpc}$ from the Sun; at most one RRab star may have been missed.

\section{SPECTROSCOPIC OBSERVATIONS}

The group of eight RR Lyrae stars detected in the Praesepe region at $86 \mathrm{kpc}$ from the Sun and described in the previous section is remarkable as it is the densest and most numerous group that we have detected at these distances in our preliminary search. However unlikely, this group could still be a chance association of eight distant stars, and line-of-sight velocities are needed to test whether the stars in this spatial group also form a moving group.

\subsection{Instrument Setup and the Observing Strategy}

The spectroscopic observations were obtained using two instruments: the Double Spectrograph (DBSP; Oke \& Gunn 1982) mounted on the Palomar $5.1 \mathrm{~m}$ telescope and the Low-Resolution Imager and Spectrograph (LRIS; Oke et al. 1995) mounted on the Keck I telescope. A 1" wide slit, a 600 line $\mathrm{mm}^{-1}$ grating, and a $5600 \AA$ dichroic were used with both instruments. This instrument setup covers a spectral range from $3800 \AA$ to $5700 \AA$ and results in resolutions of $R=1360$ and $R=1760$ for blue channels of DBSP and LRIS, respectively.

The target RR Lyrae stars were observed over the course of several nights in sets of one or two consecutive exposures followed by observations of FeAr (DBSP) or HgCdZn (LRIS) arc lamps. In order to avoid the discontinuity in the radial velocity curve near the maximum light, the observations were scheduled to target stars between phases of 0.1 and 0.85 of their pulsation cycle, with earlier phases being preferred as the stars are brighter then.

In addition, on one night we also targeted an RRab star (AV Peg) previously observed by Layden (1994). This star was observed in order to validate the metallicity and systemic velocity measuring procedures described below. The log of spectroscopic observations is provided in Table 2.

\subsection{Data Reduction and Calibration}

All data were reduced with standard IRAF ${ }^{12}$ routines, and spectra were extracted using an optimal (inverse varianceweighted) method (Horne 1986). The spectra of objects observed in two exposures were reduced and calibrated independently and then combined. The wavelength calibration of DBSP spectra was done using a single set of $49 \mathrm{FeAr}$ lines, while the

\footnotetext{
12 http://iraf.noao.edu
} 
Table 2

Observing Log, Velocities, and Metallicities

\begin{tabular}{|c|c|c|c|c|c|c|c|c|}
\hline ID $^{\mathrm{a}}$ & $\begin{array}{l}\text { HJD }_{\text {spectrum }}{ }^{\mathrm{b}} \\
\text { (d) }\end{array}$ & $\begin{array}{c}\text { Exposures }^{c} \\
\text { (s) }\end{array}$ & Phase $^{\mathrm{d}}$ & $\begin{array}{c}v_{r} \pm \sigma_{\mathrm{cc}} \pm \sigma_{\mathrm{zpt}} \mathrm{e} \\
\left(\mathrm{km} \mathrm{s}^{-1}\right)\end{array}$ & $\begin{array}{l}v_{\gamma} \pm \sigma_{\gamma}^{\mathrm{f}} \\
\left(\mathrm{km} \mathrm{s}^{-1}\right)\end{array}$ & $\begin{array}{c}\left\langle v_{\gamma}\right\rangle^{\mathrm{g}} \\
\left(\mathrm{km} \mathrm{s}^{-1}\right)\end{array}$ & $\begin{array}{c}\left\langle v_{\mathrm{gsr}}\right\rangle^{\mathrm{h}} \\
\left(\mathrm{km} \mathrm{s}^{-1}\right)\end{array}$ & $\begin{array}{l}{[\mathrm{Fe} / \mathrm{H}]^{\mathrm{i}}} \\
\quad(\mathrm{dex})\end{array}$ \\
\hline RR1 & 55866.028827 & $2 \times 1200$ & 0.38 & $151.2 \pm 8.6 \pm 4.0$ & $165.5 \pm 15.5$ & $159.9 \pm 8.5$ & $75.6 \pm 8.5$ & -1.5 \\
\hline RR1 & 55952.995976 & $1 \times 1800^{*}$ & 0.17 & $115.8 \pm 4.8 \pm 3.2$ & $155.2 \pm 15.4$ & & & -1.7 \\
\hline RR2 & 55865.996308 & $2 \times 1200$ & 0.18 & $117.8 \pm 6.8 \pm 5.7$ & $156.0 \pm 16.7$ & $156.0 \pm 16.7$ & $69.1 \pm 16.7$ & -0.9 \\
\hline RR3 & 55917.913149 & $2 \times 1800$ & 0.42 & $155.0 \pm 4.2 \pm 6.9$ & $164.6 \pm 14.6$ & $157.0 \pm 9.8$ & $79.9 \pm 9.8$ & -1.5 \\
\hline RR4 & 55916.996225 & $2 \times 1800$ & 0.49 & $181.5 \pm 9.8 \pm 10.9$ & $182.7 \pm 18.9$ & $182.7 \pm 18.9$ & $93.9 \pm 18.9$ & -1.4 \\
\hline RR5 & 55895.934069 & $2 \times 1200$ & 0.84 & $129.5 \pm 7.1 \pm 16.8$ & $98.2 \pm 23.3$ & $91.3 \pm 12.4$ & $6.5 \pm 12.4$ & -2.6 \\
\hline RR5 & 55922.944423 & $2 \times 1800$ & 0.11 & $47.9 \pm 4.9 \pm 7.7$ & $94.5 \pm 17.7$ & & & -2.4 \\
\hline RR5 & 55926.018096 & $2 \times 1800$ & 0.15 & $34.2 \pm 7.5 \pm 19.9$ & $76.0 \pm 25.8$ & & & -2.5 \\
\hline RR6 & 55926.967532 & $1 \times 1800^{*}$ & 0.60 & $117.3 \pm 5.4 \pm 2.5$ & $105.4 \pm 13.6$ & $105.4 \pm 13.6$ & $18.1 \pm 13.6$ & -2.1 \\
\hline RR7 & 55917.864566 & $2 \times 1800$ & 0.39 & $87.5 \pm 3.9 \pm 9.9$ & $100.7 \pm 16.2$ & $100.7 \pm 16.2$ & $23.0 \pm 16.2$ & -2.2 \\
\hline RR8 & 55888.997603 & $2 \times 1800$ & 0.39 & $88.1 \pm 8.2 \pm 2.2$ & $101.3 \pm 14.9$ & $101.3 \pm 14.9$ & $22.4 \pm 14.9$ & -1.2 \\
\hline RR9 & 56037.732088 & $2 \times 1800$ & 0.50 & $109.8 \pm 16.8 \pm 3.2$ & $109.8 \pm 17.1$ & $109.8 \pm 20.9$ & $38.0 \pm 20.9$ & -2.0 \\
\hline RR10 & 56037.175892 & $2 \times 1800$ & 0.44 & $152.1 \pm 11.8 \pm 5.7$ & $159.3 \pm 13.1$ & $159.3 \pm 17.8$ & $88.6 \pm 17.8$ & -1.9 \\
\hline AV Peg & 55917.604262 & $1 \times 270$ & 0.41 & $-75.5 \pm 7.8 \pm 11.9$ & $-64.8 \pm 14.2$ & $-64.8 \pm 14.2$ & $135.1 \pm 14.2$ & 0.1 \\
\hline
\end{tabular}

Notes.

${ }^{a}$ Short name.

${ }^{b}$ Reduced Heliocentric Julian Date when the spectrum was taken (HJD -2,400,000).

c Exposure times (“*” denotes Keck I/LRIS setup).

d Pulsation phase when the spectrum was taken.

e Radial velocity (not corrected for pulsations) and its uncertainties (cross-correlation and zero point).

${ }^{\mathrm{f}}$ Systemic (center-of-mass) velocity (corrected for pulsations) and its uncertainties (cross-correlation, zero point, and model errors added in quadrature).

$\mathrm{g}$ Weighted average of multiple heliocentric systemic velocities and its uncertainty.

${ }^{\mathrm{h}}$ Galactocentric rest-frame velocity and its uncertainty.

${ }^{\mathrm{i}}$ Metallicity.

LRIS spectra were calibrated using a set of $13 \mathrm{HgCdZn}$ lines. The highest rms scatter of the fit of the wavelength calibration was $0.1 \AA\left(\sim 6 \mathrm{~km} \mathrm{~s}^{-1}\right.$ at $\left.4750 \AA\right)$ for DBSP spectra and $0.05 \AA$ ( $\sim 3 \mathrm{~km} \mathrm{~s}^{-1}$ at $4750 \AA$ ) for LRIS spectra.

To correct for the possible wavelength shift in DBSP spectra during an exposure (e.g., due to instrument flexure), we measure the wavelengths $\left(\lambda_{\text {obs }}\right)$ of three $[\mathrm{Hg} \mathrm{I}]$ sky lines and offset the wavelength zero point by the average of $\Delta \lambda=$ $\lambda_{\text {obs }}-\lambda_{\text {lab }}$, where $\lambda_{\text {lab }}=4046.565,4358.335$, and $5460.750 \AA$ are the laboratory wavelengths of $[\mathrm{Hg} \mathrm{I}]$ sky lines when observed in air. The uncertainty in the wavelength zero point ( $\sigma_{\text {wave }}$ in units of $\AA$ ) is estimated using the standard deviation of $\Delta \lambda$.

To correct LRIS spectra, we use the [O I] sky line at $\lambda_{\text {lab }}=$ $5577.34 \AA$. As this line is outside the range of arc lines used to calibrate LRIS spectra (the reddest arc line is at $\sim 5460 \AA$ ), it is possible its position, and therefore the wavelength zero point of LRIS spectra, may be more uncertain if the shape of the wavelength solution near $5577.34 \AA$ changes significantly throughout the night. This would correspond to a distortion of the LRIS focal plane, not to flexure, which is essentially a differential offset along this plane. To estimate the uncertainty in the shape of the wavelength solution near $5577.34 \AA$, we use the following procedure. First, we evaluate on a fixed grid all wavelength solutions obtained from arc images taken during a night. The solutions are offset to the same wavelength zero point by subtracting the average difference between a solution and some reference solution. Then, we evaluate all solutions at a single grid point for which the reference solution returns $5577.34 \AA$. We find the rms scatter around this point (5577.34 $\AA$ ) to be very similar to the rms scatter of the fit of wavelength solutions $\left(\sim 0.05 \AA\right.$ or $\left.2-3 \mathrm{~km} \mathrm{~s}^{-1}\right)$. This means that the shape of the wavelength solution near $5577.34 \AA$ does not vary significantly, even though this region is outside the range of arc lines used in wavelength calibration. Therefore, for LRIS spectra we adopt the rms scatter of the fit of the wavelength calibration as the uncertainty in the wavelength zero point $\left(\sigma_{\text {wave }} \sim 0.05 \AA\right)$. Later in Section 5.3, we will use $\sigma_{\text {wave }}$ (in units of $\AA$ ) to estimate the zero-point uncertainty in measured radial velocities $\left(\sigma_{\text {zpt }}\right.$ in units of $\left.\mathrm{km} \mathrm{s}^{-1}\right)$.

The $\mathrm{S} / \mathrm{N}$ of the final spectra at $4750 \AA$ ranged from 10 to 20 for DBSP spectra and 20 to 30 for LRIS spectra. Figure 3 shows a few examples of observed DBSP spectra, ordered from the highest to lowest $\mathrm{S} / \mathrm{N}$. The Balmer lines (starting at $\mathrm{H} \beta$ ) and [Ca II] $\mathrm{H}$ and $\mathrm{K}$ lines are easily seen in the spectra. The $\mathrm{CH}$ G-band absorption feature at $4300 \AA$ is relatively weak in spectra of RR Lyrae stars, indicating that the RR Lyrae stars shown in Figure 3 are likely more metal-poor than the ELODIE template star HD $22879([\mathrm{Fe} / \mathrm{H}]=-0.84$ dex $)$. The ELODIE template star HD 22879 is an F9 dwarf $\left(T_{\text {eff }}=5841 \mathrm{~K}\right)$ and is one of the radial velocity standard stars used in the next section.

\subsection{Line-of-sight Velocities}

Heliocentric radial velocities, $v_{r}$, were obtained by crosscorrelating our spectra with spectra of $11 \mathrm{~F} 2$ to F9 dwarf stars (hereafter template spectra: see Table 3) taken from the ELODIE catalog $^{13}$ (Moultaka et al. 2004). The template spectra were degraded to the resolution of our observations and were selected to encompass the range of spectral types covered by RRab stars as they pulsate. Expanding the list of template spectra to include more stars and more spectral types did not significantly reduce the uncertainty of measured radial velocities.

\footnotetext{
13 http://atlas.obs-hp.fr/elodie/
} 


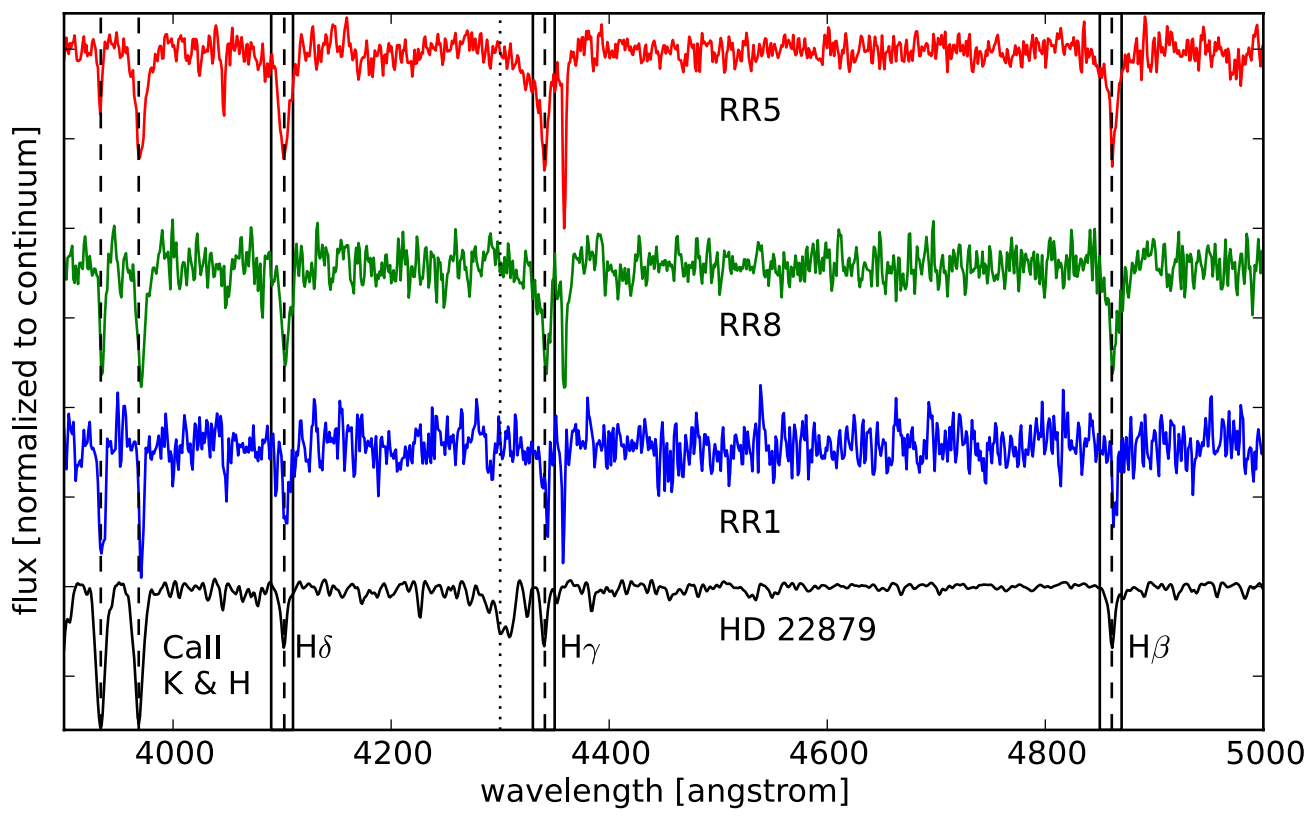

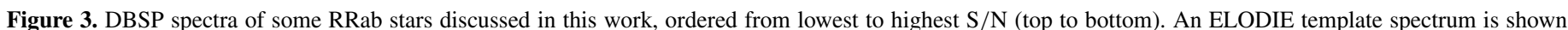

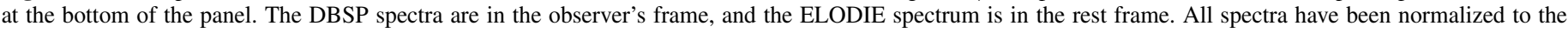

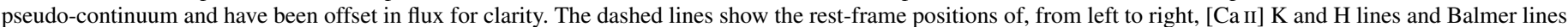

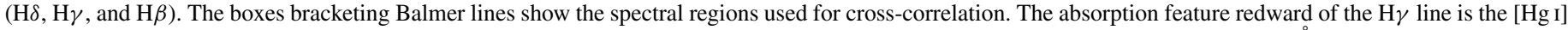

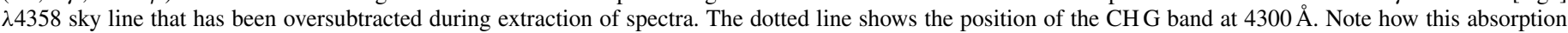

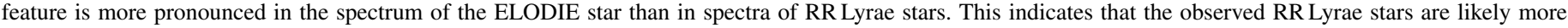
metal-poor than the ELODIE star (an F9 dwarf star, with $[\mathrm{Fe} / \mathrm{H}]=-0.84 \mathrm{dex}$ and $T_{\mathrm{eff}}=5841 \mathrm{~K}$ ).

(A color version of this figure is available in the online journal.)

Table 3

ELODIE Template Stars

\begin{tabular}{lcc}
\hline \hline Name & ELODIE ID Number & Spectral Type \\
\hline HD 49933 & 00282 & F2V \\
HD 140283 & 00480 & sdF3 \\
HD 102870 & 00414 & F8V \\
HD 693 & 00004 & F5V \\
HD 7476 & 00690 & F5V \\
HD 13555 & 00998 & F5V \\
HD 3268 & 00010 & F7V \\
HD 222368 & 00855 & F7V \\
HD 19994 & 00084 & F8V \\
HD 22484 & 00092 & F9V \\
HD 22879 & 00096 & F9V \\
\hline
\end{tabular}

The cross-correlation was performed using the FXCOR task in IRAF, with template and science spectra normalized to the pseudo-continuum prior to cross-correlation. For our relatively low resolution spectra, the narrowest cross-correlation peaks were obtained by restricting the correlation region in template and science spectra to cores and wings of Balmer lines (spectral regions used in cross-correlation are shown in Figure 3). Addition of other spectral regions or Fourier filtering did not significantly improve the cross-correlation.

The radial velocities were measured by fitting a Gaussian to the top $25 \%$ of the cross-correlation peak, and the final $v_{r}$ radial velocity of an object was calculated as the average of the $11 v_{r}$ values obtained from 11 cross-correlations with ELODIE templates, where each $v_{r}$ value was weighted by its crosscorrelation error $\sigma_{\mathrm{cc}}$. The cross-correlation error $\sigma_{\mathrm{cc}}$ reported by FXCOR includes the uncertainties due to broadening of spectral features during an exposure, random errors in wavelength calibration, and errors due to ELODIE template mismatch.
Therefore, radial velocities from better template fits contribute more toward the final radial velocity value.

In addition to the cross-correlation error, there is an error in radial velocity due to the uncertainty in determining the wavelength zero point. We calculate this zero-point error as

$$
\sigma_{\mathrm{zpt}}=\frac{\sigma_{\mathrm{wave}} c}{4750}
$$

in units of $\mathrm{km} \mathrm{s}^{-1}$, where $\sigma_{\text {wave }}$ is the uncertainty in wavelength zero point in units of $\AA$ (see Section 5.2). The final $v_{r}$ radial velocities and their uncertainties (cross-correlation and zero point) are listed in Table 2.

Following Hawley \& Barnes (1985), the systemic (centerof-mass) velocity of RRLyrae stars, $v_{\gamma}$, was obtained by fitting the radial velocity curve of the RRab star X Ari to observed heliocentric velocities. We used Layden's (1994) parameterization (see his Figure 8 ) of the radial velocity curve that Oke (1966) measured from the $\mathrm{H} \gamma$ line. This template is an appropriate choice for fitting radial velocities measured using Balmer lines (i.e., as done in this work). Radial velocity templates based on measurements of metallic lines, such as the one presented in Liu (1991), would not be appropriate here as radial velocity curves of metallic and Balmer lines have different shapes and amplitudes (Oke 1966). The X Ari template was shifted in velocity to match the measurement for each star at the corresponding phase, and the systemic velocity is that of the shifted template at phase 0.5. Two examples of template fits are shown in Figure 4.

Finally, the error calculation for the systemic velocity includes terms that take into account errors in the model radial velocity curve. Following Vivas et al. (2005, see their Section 3), we use the expression given below in which the third term accounts for likely uncertainties in the phase where the velocity curve passes 


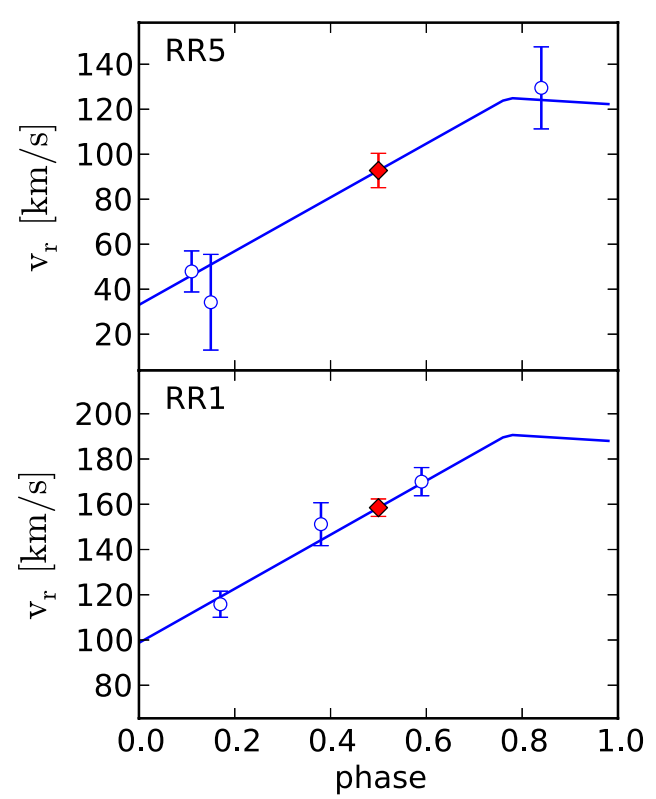

Figure 4. Fits of the radial velocity template of X Ari (solid line) to two stars in our sample. The open circles show individual $v_{r}$ measurements, and their error bars indicate the uncertainty (cross-correlation and zero-point errors added in quadrature). The red diamond symbol at phase 0.5 shows the systemic velocity calculated as the weighted average of $v_{\gamma}$ values, where the $v_{\gamma}$ values were obtained by fitting the model template to each of the observed radial velocities. The uncertainty in the systemic velocity includes the observational errors (crosscorrelation and zero point) and model errors.

(A color version of this figure is available in the online journal.)

through the systemic velocity, and the fourth term is related to possible variations in the slope of the velocity curve:

$$
\sigma_{\gamma}^{2}=\sigma_{\mathrm{cc}}^{2}+\sigma_{\mathrm{zpt}}^{2}+(119.5 \times 0.1)^{2}+(23.9 \Delta \phi)^{2},
$$

where $\Delta \phi=\phi_{\mathrm{obs}}-0.5$ and $\phi_{\mathrm{obs}}$ is the phase of observation. The $\sigma_{\mathrm{cc}}$ and $\sigma_{\mathrm{zpt}}$ are the cross-correlation and zero-point error, respectively. The contributions of model uncertainties are of the order of $\sim 15 \mathrm{~km} \mathrm{~s}^{-1}$ and are higher than the observed radial velocity errors ( $\sigma_{\mathrm{cc}}$ and $\sigma_{\mathrm{zpt}}$ added in quadrature). Therefore, to reduce the uncertainty in the systemic velocity, we need to reduce the uncertainty introduced by the model, that is, we should observe the radial velocity curve more than once.

When there is more than one $v_{\gamma}$ estimate available for an RRab star due to multiple measurements, we simply calculate the weighted average of $v_{\gamma},\left\langle v_{\gamma}\right\rangle$, and its associated uncertainty. Finally, we correct the heliocentric systemic velocities $\left\langle v_{\gamma}\right\rangle$ for the solar motion with respect to the Galactic center using

$$
\begin{aligned}
\left\langle v_{\mathrm{gsr}}\right\rangle= & \left\langle v_{\gamma}\right\rangle+v_{U} \cos b \cos l+\left(v_{V}+v_{\mathrm{LSR}}\right) \cos b \sin l \\
& +v_{W} \sin b,
\end{aligned}
$$

where $\left(v_{U}, v_{V}, v_{W}\right)=(10.0,5.2,7.2) \mathrm{km} \mathrm{s}^{-1}$ and $v_{\mathrm{LSR}}=$ $220 \mathrm{~km} \mathrm{~s}^{-1}$ (Binney \& Merrifield 1998), where $v_{\mathrm{LSR}}$ is the velocity of the local standard of rest. These velocities in the Galactic rest frame $\left(\left\langle v_{\mathrm{gsr}}\right\rangle\right)$ are adopted in the rest of this paper.

To validate the above procedure, we measured the radial velocity of AV Peg. The light-curve parameters for this star were taken from the GEOS RR Lyrae database (Le Borgne 2007). After correcting the observed heliocentric velocity for pulsation using the model radial velocity curve, we obtained $\left\langle v_{\gamma}\right\rangle=-64.8 \pm 18.7 \mathrm{~km} \mathrm{~s}^{-1}$. This value agrees within the uncertainties with the $\left\langle v_{\gamma}\right\rangle=-58 \pm 1 \mathrm{~km} \mathrm{~s}^{-1}$ estimated by Layden (1994, see his Table 9).
Table 4

Equivalent-width Standard Stars

\begin{tabular}{lcccc}
\hline \hline Name & $\begin{array}{c}W^{\prime}(\mathrm{K}) \\
(\text { mean } \pm \mathrm{sd})\end{array}$ & $\begin{array}{c}W^{\prime}(\mathrm{H} \delta) \\
(\mathrm{mean} \pm \mathrm{sd})\end{array}$ & $\begin{array}{c}W^{\prime}(\mathrm{H} \gamma) \\
(\text { mean } \pm \mathrm{sd})\end{array}$ & $\begin{array}{c}W^{\prime}(\mathrm{H} \beta) \\
(\text { mean } \pm \mathrm{sd})\end{array}$ \\
\hline Kopff 27 & $1.72 \pm 0.06$ & $10.5 \pm 0.4$ & $10.2 \pm 0.3$ & $8.84 \pm 0.05$ \\
HD 180482 & $2.42 \pm 0.07$ & $10.26 \pm 0.09$ & $9.2 \pm 0.1$ & $8.7 \pm 0.1$ \\
BD +25 1981 & $2.79 \pm 0.05$ & $5.0 \pm 0.2$ & $5.4 \pm 0.3$ & $5.12 \pm 0.03$ \\
HD 155967 & $6.6 \pm 0.2$ & $3.0 \pm 0.3$ & $3.89 \pm 0.08$ & $3.95 \pm 0.09$ \\
HD 112299 & $8.4 \pm 0.2$ & $1.9 \pm 0.2$ & $2.10 \pm 0.07$ & $3.02 \pm 0.06$ \\
\hline Kopff 27 & 1.89 & 9.68 & 10.44 & 8.89 \\
BD +25 1981 & 2.90 & 5.47 & 6.05 & 5.08 \\
HD 155967 & 6.90 & 2.75 & 3.84 & 4.05 \\
HD 112299 & 8.97 & 1.64 & 2.10 & 2.98 \\
\hline
\end{tabular}

Note. The horizontal line separates equivalent-width standard stars observed with DBSP (over three nights) and LRIS (over a single night).

\subsection{Metallicities}

Spectroscopic metallicities were measured following the method and calibration of Layden (1994), which involves comparing the pseudo-equivalent width of the [Ca II] $\mathrm{K}$ line, $W(\mathrm{~K})$, against the mean pseudo-equivalent widths of $\beta, \gamma$, and $\delta$ Balmer lines, $W(\mathrm{H})$.

The pseudo-equivalent widths of the [Ca II] K line and Balmer lines were measured using the EWIMH program ${ }^{14}$ (written by A. Layden) from DBSP and LRIS spectra normalized to the pseudo-continuum. Measured pseudo-equivalent widths $\left(W^{\prime}(\mathrm{K}), W^{\prime}(\mathrm{H} \delta), W^{\prime}(\mathrm{H} \gamma)\right.$, and $\left.W^{\prime}(\mathrm{H} \beta)\right)$ were transformed to the Layden (1994) pseudo-equivalent-width system using

$$
\begin{gathered}
W(\mathrm{~K})=1.23 W^{\prime}(\mathrm{K})-0.44 \\
W(\mathrm{H} \delta)=0.84 W^{\prime}(\mathrm{H} \delta)+1.64 \\
W(\mathrm{H} \gamma)=1.13 W^{\prime}(\mathrm{H} \gamma)-0.63 \\
W(\mathrm{H} \beta)=1.02 W^{\prime}(\mathrm{H} \beta)+0.98
\end{gathered}
$$

for pseudo-equivalent widths measured from DBSP spectra and using

$$
\begin{gathered}
W(\mathrm{~K})=1.16 W^{\prime}(\mathrm{K})-0.39 \\
W(\mathrm{H} \delta)=1.02 W^{\prime}(\mathrm{H} \delta)+1.39 \\
W(\mathrm{H} \gamma)=1.07 W^{\prime}(\mathrm{H} \gamma)-0.45 \\
W(\mathrm{H} \beta)=1.01 W^{\prime}(\mathrm{H} \beta)+1.18
\end{gathered}
$$

for pseudo-equivalent widths measured from LRIS spectra. To derive these equations, we observed five of the equivalentwidth standard stars of Layden (1994) with DBSP and four more with LRIS (see Table 4). Comparing our measurements of their pseudo-equivalent widths with those listed in Layden (1994) yielded Equations (10)-(17). After the transformation, the $W(\mathrm{~K})$ was corrected for interstellar [Ca II] absorption using

\footnotetext{
${ }^{14}$ http://physics.bgsu.edu/ layden/ASTRO/DATA/EXPORT/EWIMH/ ewimh.htm
} 
the Beers (1990) model. This correction decreases the measured metallicities by $\sim 0.15$ dex.

The spectroscopic metallicity was calculated as

$$
[\mathrm{Fe} / \mathrm{H}]=\frac{W(\mathrm{~K})-a-b W(\mathrm{H})}{c+d W(\mathrm{H})},
$$

where $a=13.858, b=-1.185, c=4.228$, and $d=-0.32$ (see Table 8 in Layden 1994). Equation (18) was obtained by inverting Equation (7) from Layden (1994). The metallicities obtained using this method are listed in Table 2.

For the most part, $[\mathrm{Fe} / \mathrm{H}]$ estimates obtained from multiple observations of the same star are consistent within $0.1-0.2$ dex, even for RR1, which was observed with different instruments (DBSP and LRIS). This scatter is consistent with the systematic uncertainty of this method, which is $\sim 0.15 \mathrm{dex}$ (Layden 1994). The exception to this is star RR3, for which we measured $[\mathrm{Fe} / \mathrm{H}]=-1.5 \mathrm{dex}$ from a DBSP spectrum and $[\mathrm{Fe} / \mathrm{H}]=-2.0 \mathrm{dex}$ from an LRIS spectrum. If we assume $[\mathrm{Fe} / \mathrm{H}]=-1.8 \mathrm{dex}$ as the metallicity of this star (the average of -1.5 and -2.0 ), we find that the two measurements are still within $2 \sigma$ of the average value, which is statistically not improbable. While it may be tempting to assign lower significance to DBSP metallicity measurements due to lower S/N of DBSP spectra (10-20 versus $>20$ for LRIS spectra), multiple metallicity measurements of RR5 argue against that. RR5 has been observed with DBSP over three nights, its spectra have $\mathrm{S} / \mathrm{N}$ in the 10-20 range, and yet the standard deviation of its metallicity measurements is $0.1 \mathrm{dex}$, consistent with the systematic uncertainty.

To validate the zero point of our metallicity scale, we measured $[\mathrm{Fe} / \mathrm{H}]$ of RRab star AV Peg observed by Layden (1994). We obtained $[\mathrm{Fe} / \mathrm{H}]=0.1 \mathrm{dex}$, while Layden (1994) cites $[\mathrm{Fe} / \mathrm{H}]=-0.1$ dex. These two values are roughly within the systematic uncertainty of this method, indicating that our metallicities are on the same zero-point system as the Layden (1994) stars (i.e., the Zinn \& West 1984 globular cluster abundance scale; Layden 1994).

\section{DISCUSSION}

In this section, we address the following questions:

1. What is the mean velocity and velocity dispersion of our sample, and how do these values compare to the literature?

2. Is the observed distribution of velocities a Gaussian or a non-Gaussian distribution?

3. Are the observed velocity groups bound or unbound systems?

4. What does the metallicity of their stars say about the progenitor(s)?

5. Are the observed velocity groups related to known halo substructures?

The galactocentric rest-frame velocities $\left\langle v_{\mathrm{gsr}}\right\rangle$ listed in Table 2 indicate that the observed RR Lyrae stars can be split into two velocity groups, one moving at $\bar{v}_{\text {gsr }}=78.0 \pm 5.6 \mathrm{~km} \mathrm{~s}^{-1}$ and the other one at $\bar{v}_{\mathrm{gsr}}=16.3 \pm 7.1 \mathrm{~km} \mathrm{~s}^{-1}$, where $\bar{v}_{\mathrm{gsr}}$ is the weighted mean of $\left\langle v_{\mathrm{gsr}}\right\rangle$ values listed in Table 2 . The uncertainty in $\bar{v}_{\text {gsr }}$ is calculated as the standard error of the weighted mean. We tentatively name these moving groups Cancer groups A and $\mathrm{B}$. The weighted mean velocity for the entire sample is $\bar{v}_{\mathrm{gsr}}=53.5 \pm 4.4 \mathrm{~km} \mathrm{~s}^{-1}$.

To estimate the velocity dispersion of these moving groups, we use the following Monte Carlo procedure. We generate
1000 sets where each set contains four mock line-of-sight velocities, $v_{\text {mock }}$ (four values because there are four RRab stars in each moving group). A $v_{\text {mock }}$ value is generated by drawing a single value from a Gaussian distribution centered on the $\left\langle v_{\text {gsr }}\right\rangle$ value of a star (e.g., $75.6 \mathrm{~km} \mathrm{~s}^{-1}$ for RR1) and having a width equal to the uncertainty on $\left\langle v_{\text {gsr }}\right\rangle$ (e.g., $8.5 \mathrm{~km} \mathrm{~s}^{-1}$ for RR1). The weighted standard deviation is calculated and stored for each set, with $v_{\text {mock }}$ values weighted by uncertainty on corresponding $\left\langle v_{\mathrm{gsr}}\right\rangle$ values listed in Table 2 . The velocity dispersion is then the average value of the distribution of 1000 weighted standard deviations, and the uncertainty of velocity dispersion is the standard deviation of this distribution. Using this procedure, we find that the two moving groups have velocity dispersions of $\sigma_{v_{\text {gsr }}}=12.4 \pm 5.0 \mathrm{~km} \mathrm{~s}^{-1}$ (group A) and $\sigma_{v_{\mathrm{gsr}}}=14.9 \pm 6.2 \mathrm{~km} \mathrm{~s}^{-1}$ (group B). The velocity dispersion of the entire sample is $\sigma_{v_{\mathrm{gsr}}}=37.7 \pm 5.0 \mathrm{~km} \mathrm{~s}^{-1}$.

The measured velocity dispersion for the full sample is inconsistent with the halo velocity dispersion profile obtained by Brown et al. (2010) using a sample of 910 distant halo stars. At $92 \mathrm{kpc}$, the extrapolation of the Brown et al. (2010) model (see their Equation (6)) would describe the velocity distribution of stars as a $68 \mathrm{~km} \mathrm{~s}^{-1}$ wide Gaussian centered on $0 \mathrm{~km} \mathrm{~s}^{-1}$. The mean velocity of our full sample is at least $10 \sigma$ away from $0 \mathrm{~km} \mathrm{~s}^{-1}$, and the velocity dispersion of our full sample is at least $5 \sigma$ lower than the velocity dispersion extrapolated from the Brown et al. (2010) model. One possible explanation of this discrepancy is that Brown et al.'s (2010) Equation (6) cannot be extrapolated outside the $16 \mathrm{kpc}<R_{\mathrm{GC}}<64 \mathrm{kpc}$ range within which the relation is assumed to be valid (Brown et al. 2010). A more likely explanation is that we are observing two moving groups with their own internal kinematics (mean velocity and velocity dispersion). If that is the case, a comparison of the predicted and measured velocity dispersion for the full sample is not a sensible thing to do.

The question of whether the distribution of velocities listed in Table 2 is a Gaussian or a non-Gaussian distribution is an important one, as deviations from normality are usually interpreted as a signature of velocity groups in the smooth halo component (Harding et al. 2001; Duffau et al. 2006; Vivas et al. 2008). To test for the presence of velocity groups in the halo, Harding et al. (2001) recommend the Shapiro \& Wilk $(1965, \mathrm{SW})$ statistical test of normality to be applied to velocity histograms. This test is sensitive to many different deviations from the Gaussian shape and does not depend on the choice of mean or dispersion of the normal distribution (see Harding et al. 2001, Section 5.1, and D’Agostino \& Stephens 1986 for more details on the test).

Following the procedure detailed in Sesar et al. (2010b, see their Section 3) that takes into account uncertainties in velocities, we use the SW test and find that there is a $37 \%$ probability that the observed sample of velocities was drawn from a single Gaussian distribution. However, the SW test does not have the ability to assess the rather peculiar nature of the shape of the observed distribution (i.e., it cannot distinguish the particular nature of non-Gaussianity). To include this information, we consider the $37 \%$ chance that the measured velocities were drawn from a Gaussian distribution and estimate the probability that this chance would produce the observed distribution seen in Figure 5. We generate 100,000 samples and for each sample draw eight velocities from a Gaussian centered on $53.5 \mathrm{~km} \mathrm{~s}^{-1}$ and $37.7 \mathrm{~km} \mathrm{~s}^{-1}$ wide (i.e., the velocity distribution of the full sample of RR Lyrae stars observed in the Cancer region). Each 


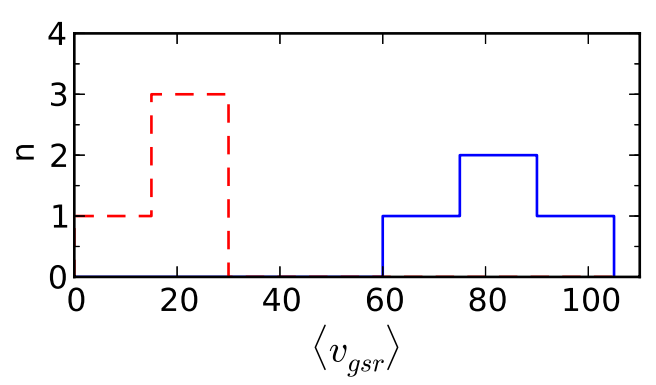

Figure 5. Histogram of $\left\langle v_{\text {gsr }}\right\rangle$ of RR Lyrae stars in Cancer groups. The bin size is $15 \mathrm{~km} \mathrm{~s}^{-1}$, which is about the average velocity error in $\left\langle v_{\mathrm{gsr}}\right\rangle$. The distribution of observed velocities seems to be bimodal, with two velocity peaks centered on $\left\langle v_{\mathrm{gsr}}\right\rangle=78 \mathrm{~km} \mathrm{~s}^{-1}$ and $\left\langle v_{\mathrm{gsr}}\right\rangle=16 \mathrm{~km} \mathrm{~s}^{-1}$, respectively. See Section 6 for a discussion of statistical significance of this bimodality.

(A color version of this figure is available in the online journal.)

sample is then divided into stars with velocities greater and smaller than $53.5 \mathrm{~km} \mathrm{~s}^{-1}$ ("positive" and "negative" groups), and the mean velocities and velocity dispersions are calculated for each group $\left(v_{\text {pos }}, v_{\text {neg }}, \sigma_{\text {pos }}\right.$, and $\left.\sigma_{\text {neg }}\right)$. In only $\sim 1.6 \%$ of generated samples, we find that the "positive" and "negative" groups have mean velocities and velocity dispersions in ranges covered by Cancer groups $\left(72<v_{\text {pos }}<84\right.$ and $7<\sigma_{\text {pos }}<18$, and $9<v_{\text {neg }}<24$ and $8<\sigma_{\text {neg }}<22$ ). The percentage of generated samples with mean velocities and velocity dispersions in ranges covered by Cancer groups is even lower $(0.02 \%)$, if the samples are drawn from a $68 \mathrm{~km} \mathrm{~s}^{-1}$ wide Gaussian centered on $0 \mathrm{~km} \mathrm{~s}^{-1}$ (i.e., the velocity distribution at $92 \mathrm{kpc}$ as extrapolated from Equation (6) by Brown et al. 2010). Therefore, even if the observed velocities were drawn from a Gaussian distribution (a 37\% chance), it is unlikely they would create the distribution seen in Figure 5, as the chance of that happening is only about $0.6 \%(0.37 \times 0.016 \sim 0.006)$, if the velocity distribution at $92 \mathrm{kpc}$ is a $37.7 \mathrm{~km} \mathrm{~s}^{-1}$ wide Gaussian centered on $53.5 \mathrm{~km} \mathrm{~s}^{-1}$, or about $0.07 \%\left(0.37 \times 0.0002 \sim 7 \times 10^{-5}\right)$, if the velocity distribution follows the extrapolation of the Brown et al. (2010) model. The next simplest explanation for the observed distribution of velocities is that we are observing two velocity groups, moving at $\bar{v}_{\text {gsr }}=78.1 \pm 5.7 \mathrm{~km} \mathrm{~s}^{-1}$ and $\bar{v}_{\mathrm{gsr}}=16.3 \pm 7.1 \mathrm{~km} \mathrm{~s}^{-1}$ and having velocity dispersions of $\sigma_{v_{\mathrm{gsr}}}=12.4 \pm 5.0 \mathrm{~km} \mathrm{~s}^{-1}$ and $\sigma_{v_{\mathrm{gsr}}}=15.0 \pm 6.1 \mathrm{~km} \mathrm{~s}^{-1}$.

These estimated velocity dispersions are consistent with velocity dispersions of dwarf spheroidal galaxies (Walker et al. 2007, e.g., Leo II) and globular clusters (Harris 1996, 2010 edition, e.g., NGC 362). Due to significant uncertainities in the velocities, these velocity dispersions are most likely upper limits on the intrinsic velocity dispersions of the Cancer groups. While the velocity dispersions of the two groups are consistent with velocity dispersions of dwarf spheroidal galaxies and globular clusters, the groups are spatially quite extended (the maximum separation between members is several kpc), making it very unlikely that they are bound systems, and more likely to be debris of tidally disrupted dwarf galaxies or globular clusters.

The metallicity of these groups may provide more information about their progenitors. The first group has a median metallicity of $[\mathrm{Fe} / \mathrm{H}]=-1.6 \mathrm{dex}$ and the second group has $[\mathrm{Fe} / \mathrm{H}]=-2.1$ dex on the Zinn \& West (1984) globular cluster abundance scale, indicating that the progenitors of these two substructures are systems with old, metal-poor populations. The metallicity spread (standard deviation) in both groups is rather large $(\sim 0.4 \mathrm{dex})$ and a few times greater than the uncertainty in individual metallicity estimates $(\sim 0.15 \mathrm{dex})$. Such a large dispersion in $[\mathrm{Fe} / \mathrm{H}]$ indicates self-enrichment and implies that the progenitor structures were dwarf galaxies and most likely not globular clusters. Using the metallicity-luminosity relation by Kirby et al. (2011, see their Equation (9)), we can place a lower limit on the luminosity of group A and B progenitors. Taking into account the intrinsic spread in this relation, we obtain $L \gtrsim 5.5 \times 10^{5} L_{\odot}$ for the group A progenitor and $L \gtrsim 9.1 \times 10^{3} L_{\odot}$ for the group B progenitor. The luminosity limit of $L \gtrsim 5.5 \times 10^{5} L_{\odot}$ indicates that the group A progenitor was likely more luminous than one of the classical dwarf spheroidal galaxies, such as Leo II (e.g., see Figure 3 by Kirby et al. 2011).

However, we do note that there are only four stars in each group and that the metallicity spread can change drastically if a single measurement is not considered when estimating the spread. For example, if the metallicity of RR8 is not taken into account when estimating the metallicity spread of the second group, the new spread would be $\sim 0.1$ dex, which would be consistent with measurement uncertainties and consistent with a single stellar population (i.e., a globular cluster as the progenitor). Additional observations of RR Lyrae stars on the metal-poor and metal-rich ends (e.g., RR2, RR3, and RR8) would be very useful as they would further test the validity of the estimated metallicity dispersions.

Sharma et al. (2010) have found two overdensities of M giants extending into this region of the sky, one at $22.6 \pm 11 \mathrm{kpc}$ (their group A13) and the other one at $96.6 \pm 48 \mathrm{kpc}$ (their group A9). Both of these groups have density peaks (centers) that are offset by at least $20^{\circ}$ from the location of Cancer groups discussed here, with the group A13 peaking at $l \sim 144^{\circ}$ and $b \sim 31^{\circ}$ and group A9 peaking at $l \sim 188^{\circ}$ and $b \sim 20^{\circ}$. The Cancer groups are likely not associated with the Sharma et al. (2010) group A13 based on estimated distances of $\mathrm{M}$ giants provided by Sharma et al. (2010; 22.6 $\pm 11 \mathrm{kpc}$, see their Table 2). Based on the distance alone $(96.6 \pm 48 \mathrm{kpc})$, it is not impossible that the Cancer groups may be associated with the Sharma et al. (2010) group A9. However, we do note that the Cancer groups discussed in this work are on the very edge of the Sharma et al. (2010) group A9 (see their Figure 7) and that the distance of group A9 has uncertainty of $\sim 50 \mathrm{kpc}$.

Newberg et al. (2003) have found an overdensity of A-type stars about $10^{\circ}$ north in declination from Cancer groups and at a similar distance ( $~ 90 \mathrm{kpc}$ from the Sun; see their Figure 1). At a similar location, Ruhland et al. (2011) have found "a very faint indication of an overdensity" of blue horizontal branch (BHB) stars. Both studies speculated that the overdensities they observed were associated with the Sagittarius tidal debris. However, recent modeling of the Sagittarius streams by Law \& Majewski (2010) suggests that there should not be any debris at these distances. It is unclear whether these overdensities and Cancer groups are related due to lack of spectroscopic follow-up of BHB and A-type stars in Newberg et al. (2003) and Ruhland et al. (2011) overdensities.

The surface density of RRab stars in Cancer groups A and B seems to be similar to that of the Pisces Overdensity/Stream $\left(\sim 0.3\right.$ RRab per $\left.\mathrm{deg}^{2}\right)$, a halo substructure located at a similar distance ( $\sim 85 \mathrm{kpc})$ from the Galactic center (Sesar et al. 2007; Watkins et al. 2009; Sesar et al. 2010a). In addition, both of these substructures seem to have two velocity groups (the Pisces Overdensity has groups moving at $50 \mathrm{~km} \mathrm{~s}^{-1}$ and $-52 \mathrm{~km} \mathrm{~s}^{-1}$; Kollmeier et al. 2009; Sesar et al. 2010b). The morphology of both substructures is reminiscent of debris clouds observed in some simulations (e.g., Bullock \& Johnston 2005; Johnston 
et al. 2008). According to a recent study by Johnston et al. (2012), the clouds represent debris that is slowly turning around at apocenters of orbits that are typically more eccentric than orbits of debris streams, such as the Sagittarius stream. If so, then "there must be low-density stellar streams moving between these apocentric clouds and passing through the inner Galaxy at high speed" (Johnston et al. 2012). By following up RR Lyrae stars selected from PTF and other surveys, it will be possible to identify such low-density streams, if they exist, and to explore their relationship with Cancer groups A and B.

\section{CONCLUSIONS}

We confirm the existence of two kinematic groups in the direction of the Cancer constellation (R.A. $\sim 129^{\circ}$ and decl. $\sim 20^{\circ}$, or $l \sim 205^{\circ}$ and $b \sim 32^{\circ}$ ), located at $92 \mathrm{kpc}$ from the Galactic center ( $86 \mathrm{kpc}$ from the Sun). These groups, tentatively named Cancer groups $\mathrm{A}$ and $\mathrm{B}$, are moving at $\bar{v}_{\mathrm{gsr}}^{A}=78.0 \pm 5.7 \mathrm{~km} \mathrm{~s}^{-1}$ (Cancer group A) and $\bar{v}_{\mathrm{gsr}}^{B}=16.3 \pm$ $3.8 \mathrm{~km} \mathrm{~s}^{-1}$ (Cancer group B). The groups have velocity dispersions smaller than $15 \mathrm{~km} \mathrm{~s}^{-1}$, are spatially extended (about several kpc), are metal-poor (median metallicities of $[\mathrm{Fe} / \mathrm{H}]^{\mathrm{A}}=-1.6 \mathrm{dex}$ and $\left.[\mathrm{Fe} / \mathrm{H}]^{\mathrm{B}}=-2.1 \mathrm{dex}\right)$, and have a metallicity spread of $\sim 0.4$ dex. These results suggest that the observed groups are debris of tidally disrupted dwarf galaxies, possibly near the apocenters of their orbits. Whether these groups are related to known substructures in the Galactic halo is unclear at this point, and answering this question may require extensive orbit modeling and comparisons with simulations (e.g., as done by Johnston et al. 2012 and Carlin et al. 2012).

Observations of two RR Lyrae stars (RR9 and RR10 in Tables 1 and 2) obtained after this paper was submitted for peer review may help with the modeling of Cancer groups' orbits. The two RR Lyrae stars have velocities of $88 \mathrm{~km} \mathrm{~s}^{-1}$ and $38 \mathrm{~km} \mathrm{~s}^{-1}$ that are consistent (to within the uncertainties) with the mean velocities of Cancer groups A and B and are at similar distances ( $\sim 76 \mathrm{kpc}$ from the Sun). They are offset $\sim 25^{\circ}$ east of the Cancer groups and may indicate an eastward extension of these groups. However, due to the sparse coverage in PTF of the sky between Cancer groups and RR Lyrae stars RR9 and RR10, we are unable to verify at this point whether RR9 and RR10 are truly related to the Cancer groups or not.

Initially, this work was motivated by a hypothesis that distant halo substructures may be found by simply looking for distant spatial groups of RR Lyrae stars. Based on this work and previous work by Kollmeier et al. (2009) and Sesar et al. (2010b), we conclude that this is indeed an efficient approach to finding and following up halo substructures. So far, all of the distant (galactocentric distances greater than $80 \mathrm{kpc}$ ) RR Lyrae stars located in spatial groups have proven to be members of a halo substructure (e.g., Cancer groups in this work and RR Lyrae stars in the Pisces Overdensity; Kollmeier et al. 2009; Sesar et al. 2010b). Since the density profile of the relatively smooth, inner halo steepens beyond $30 \mathrm{kpc}$ (Sesar et al. 2011; Deason et al. 2011), it may be that the majority, if not all, of RR Lyrae stars beyond $\sim 30-40 \mathrm{kpc}$ are part of some halo substructure. Spectroscopic follow-up of distant RR Lyrae stars not studied in this work may provide more data to support or refute this hypothesis, and we plan to follow this strategy with other RR Lyrae stars observed by the PTF.

J.G.C. and B.S. thank NSF grant AST-0908139 to J.G.C for partial support, as do S.R.K (to NSF grant AST-1009987) and
C.J.G (for a NASA grant). Support for this work was provided by NASA through Hubble Fellowship grant 51256.01 awarded to E.N.K by the Space Telescope Science Institute, which is operated by the Association of Universities for Research in Astronomy, Inc., for NASA, under contract NAS 5-26555.

We thank the referee for a thorough review and suggestions that led to an improved manuscript. B.S. thanks Ž. Ivezić, B. Willman, and K. Vivas for useful discussions. We thank I. Arcavi, A. Gal-Yam, P. Groot, A. Horesh, and D. Perley for observing at Keck and Palomar. We thank the staff at the Palomar Hale telescope for help and support with observations. We are grateful to the many people who have worked to make the Keck Telescope and its instruments a reality and to operate and maintain the Keck Observatory. The authors wish to extend special thanks to those of Hawaiian ancestry on whose sacred mountain we are privileged to be guests. Without their generous hospitality, none of the observations presented herein would have been possible.

This article is based on observations obtained with the Samuel Oschin Telescope as part of the Palomar Transient Factory project, a scientific collaboration between the California Institute of Technology, Columbia University, Las Cumbres Observatory, the Lawrence Berkeley National Laboratory, the National Energy Research Scientific Computing Center, the University of Oxford, and the Weizmann Institute of Science.

\section{REFERENCES}

Agüeros, M. A., Covey, K. R., Lemonias, J. J., et al. 2011, ApJ, 740, 110 Aihara, H., Allende Prieto, C., An, D., et al. 2011, ApJS, 193, 29

Beers, T. C. 1990, AJ, 99, 323

Belokurov, V., Zucker, D. B., Evans, N. W., et al. 2007, ApJ, 654, 897

Binney, J., \& Merrifield, M. (ed.) 1998, in Galactic Astronomy (Princeton, NJ: Princeton Univ. Press)

Brown, W. R., Geller, M. J., Kenyon, S. J., \& Diaferio, A. 2010, AJ, 139, 59

Bullock, J. S., \& Johnston, K. V. 2005, ApJ, 635, 931

Carlin, J. L., Yam, W., Casetti-Dinescu, D., et al. 2012, ApJ, 753, 145

Chaboyer, B. 1999, in Post-Hipparcos Cosmic candles, ed. A. Heck \& F. Caputo (Dordrecht: Kluwer), 111

D’Agostino, R. B., \& Stephens, M. A. 1986, Goodness of Fit Techniques (New York: Marcel Dekker)

Deason, A. J., Belokurov, V., \& Evans, N. W. 2011, MNRAS, 416, 2903

Duffau, S., Zinn, R., Vivas, A. K., et al. 2006, ApJ, 636, L97

Friedman, J. H. 1984, A Variable Span Smoother, LCS Technical Report 5

Grillmair, C. J. 2006, ApJ, 645, 37L

Grillmair, C. J., \& Dionatos, O. 2006, ApJ, 643, 17

Grillmair, C. J., Laher, R., Surace, J., et al. 2010, in ASP Conf. 434, Astronomical Data Analysis Software and Systems XIX, ed. Y. Mizumoto, K.-I. Morita, \& M. Ohishi (San Francisco, CA: ASP), 28

Harding, P., Morrison, H. L., Olszewski, E. W., et al. 2001, AJ, 122, 1397

Harris, W. E. 1996, AJ, 112, 1487

Hawley, S. L., \& Barnes, T. G., III. 1985, PASP, 97, 551

Honeycutt, R. K. 1992, PASP, 104, 435

Horne, K. 1986, PASP, 98, 609

Ibata, R. A., Gilmore, G., \& Irwin, M. J. 1994, Nature, 370, 194

Johnston, K. V., Bullock, J. S., Sharma, S., et al. 2008, ApJ, 689, 936

Johnston, K. V., Sheffield, A. A., Majewski, S. R., \& Sharma, S. 2012, arXiv:1202.5311

Jurić, M. 2011, BAAS, 43, 433.19

Kirby, E. N., Lanfranchi, G. A., Simon, J. D., Cohen, J. G., \& Guhathakurta, P. 2011, ApJ, 727, 78

Klypin, A., Kravtsov, A. V., Valenzuela, O., \& Prada, F. 1999, ApJ, 522, 82

Kollmeier, J. A., Gould, A., Shectman, S., et al. 2009, ApJ, 705, L158

Law, D. R., \& Majewski, S. R. 2010, ApJ, 714, 229

Law, N. M., Kulkarni, S. R., Dekany, R. G., et al. 2009, PASP, 121, 1395

Layden, A. C. 1994, AJ, 108, 1016

Le Borgne, J. F., Paschke, A., Vandenbroere, J., et al. 2007, A\&A, 476, 307

Levitan, D., Fulton, B. J., Groot, P. J., et al. 2011, ApJ, 739, 68

Liu, T. 1991, PASP, 103, 205

Lupton, R. H., Ivezić, Ž., Gunn, J. E., et al. 2002, Proc. SPIE, 4836, 350 
Majewski, S. R., Skrutskie, M. F., Weinberg, M. D., \& Ostheimer, J. C. 2003, ApJ, 599, 1082

Moore, B., Ghigna, S., Governato, F., et al. 1999, ApJ, 524, 19

Moultaka, J., Ilovaisky, S. A., Prugniel, P., \& Soubiran, C. 2004, PASP, 116, 693

Newberg, H. J., Yanny, B., Grebel, E. K., et al. 2003, ApJ, 596, L191

Ofek, E. O., Frail, D. A., Breslauer, B., et al. 2011, ApJ, 740, 65

Ofek, E. O., Laher, R., Law, N., et al. 2012, PASP, 124, 62

Oke, J. B. 1966, ApJ, 145, 468

Oke, J. B., Cohen, J. G., Carr, M., et al. 1995, PASP, 107, 375

Oke, J. B., \& Gunn, J. E. 1982, PASP, 94, 586

Polishook, D., Ofek, E. O., Waszczak, A., et al. 2012, MNRAS, 421, 2094

Rahmer, G., Smith, R., Velur, V., et al. 2008, Proc. SPIE, 7014, 163

Rau, A., Kulkarni, S. R., Law, N. M., et al. 2009, PASP, 121, 1334

Reimann, J. D. 1994, PhD thesis, Univ. California, Berkeley

Ruhland, C., Bell, E. F., Rix, H.-W., \& Xue, X.-X. 2011, ApJ, 731, 119

Schlegel, D., Finkbeiner, D. P., \& Davis, M. 1998, ApJ, 500, 525
Sesar, B., Ivezić, Ž., Grammer, S. H., et al. 2010a, ApJ, 708, 717

Sesar, B., Ivezić, Ž., Lupton, R. H., et al. 2007, AJ, 134, 2236

Sesar, B., Jurić, M., \& Ivezić, Ž. 2011, ApJ, 731, 4

Sesar, B., Vivas, A. K., Duffau, S., \& Ivezić, Ž. 2010b, ApJ, 717, 133

Shapiro, S. S., \& Wilk, M. B. 1965, Biometrika, 52, 591

Sharma, S., Johnston, K. V., Majewski, S. R., et al. 2010, ApJ, 722, 750

Skrutskie, M. F., Cutri, R. M., Stiening, R., et al. 2006, AJ, 131, 1163

Smith, H. A. 1995, RR Lyrae Stars (Cambridge: Cambridge Univ. Press)

Vivas, A. K., Jaffé, Y. L., Zinn, R., et al. 2008, AJ, 136, 1645

Vivas, A. K., Zinn, R., \& Gallart, C. 2005, AJ, 129, 189

Walker, M. G., Mateo, M., Olszewski, E. W., et al. 2007, ApJ, 667, L53

Watkins, L. L., Evans, N. W., \& An, J. H. 2010, MNRAS, 406, 264

Watkins, L. L., Evans, N. W., Belokurov, V., et al. 2009, MNRAS, 398, 1757

Willman, B., Dalcanton, J. J., Martinez-Delgado, D., et al. 2005, ApJ, 626, L85

York, D. G., Adelman, J., Anderson, J. E., et al. 2000, AJ, 120, 1579

Zacharias, N., Finch, C., Girard, T., et al. 2010, AJ, 139, 2184

Zinn, R., \& West, M. J. 1984, ApJS, 55, 45 